\title{
The Intelligent Cosmos
}

\author{
Berov GL* \\ Engineer, Independent Innovative Ideas Researcher, Bulgaria
}

*Corresponding author: Berov G Lyubomir, Engineer, Independent Innovative Ideas Researcher, Smolyan 4700, Bulgaria, Tel: +359301-81433; Email: my_kaly1@mbox.contact.bg

\section{Editorial}

Volume 4 Issue 4

Received Date: June 15, 2020

Published Date: July 06, 2020

DOI: $10.23880 /$ jenr-16000205

\section{Abstract}

In this article, I propose a new hypothesis which offers a novel interpretation of the anthropic principle and argues against the premise that the Universe is "configured" (via its characteristic constants) specifically so that human life can exist.

Keywords: Anthropic Principle, Universe, Humanity, Dark Energy, Dark Matter, Big Bang Theory, Origin of Universe and Human Life

\section{Editorial}

Dear reader, what do you think? Could we help the modern physics a little bit via a peculiar hypothesis that provides a new explanation of the anthropic principle?

The anthropic principle is used by many world-renowned physicists to explain some of the fundamental physical constants of our universe. This principle is the philosophical premise that any data we collect about the universe is filtered by the fact that, in order for it to be observable at all, the universe must have been compatible with the emergence of conscious and sapient life that observes it. In other words scientific observation of the universe would not even be possible if the laws of the universe had been incompatible with the development of sapient life. It explains why this universe has the age and the fundamental physical constants we observe - for example the gravitational constant, the charge and mass of the electron, the mass of the proton and others, necessary to accommodate conscious life [1].

In simple words, the anthropic principle is based on the premise that everything around us is created only so that we, humans, can exist and create our messes. Here! Time and time again, man comes first. I believe that with my new hypothesis, everything can get a much more meaningful explanation [2].

So, dear reader, let's start with a brief history of our universe. According to the Big Bang theory, which is prevalent among the scientific community, the expansion of the universe begins with an extremely hot and dense phase called the Planck era, where all the mass and energy of the observable universe was concentrated in a very tiny space. From that moment on, the universe has expanded, reaching its present state. Several independent experiments confirm the theoretical formulations of the Big Bang theory. According to those experiments, the universe will continue to expand indefinitely [3].

The universe is very old, and both the universe itself, and our perception of it continue to evolve. The most accurate approximation of the age of the universe, based on observations of relic radiation, is 13.7 billion years. The universe is constantly changing, and the state of the universe today is different from any other state in its history. For example, the number of quasars and galaxies is changing and the galactic space itself is expanding [4].

But what is the universe made of? Its smallest component is the ordinary matter. The ordinary matter consists of us and all life on Earth, the planets, the stars, the galaxies. This matter is approximately 4 percent of the composition of the universe. Then, about 23 percent of the universe is the invisible (dark) matter. "Invisible" means that it does not interact with any form of radiation, including the electromagnetic radiation. It does not emit in the electromagnetic spectrum. It does not reflect either. Simply, the invisible matter does not interact with the electromagnetic spectrum, which is the main tool 


\section{Journal of Ecology and Natural Resources}

we use to observe matter. So how do we know dark matter exists? We know this because of its gravitational effect. In fact, dark matter dominates on a large scale the gravitational effects in the universe, and there is clear evidence to prove this. One example is the speed at which stars orbit galaxies. This speed is too high, and the reason is that the galaxies are covered by dark matter. The speed at which galaxies orbit within a cluster is also too high for the same reason [5].

The third major ingredient of the universe is the dark energy which comprises about 73 percent. Its existence has been confirmed both experimentally and theoretically. Experimentally, the existence of dark energy was confirmed through the discovery of the rate of expansion of the universe. Recent studies confirm previous results that the properties of dark energy have not changed over billions of years. Theoretically, the confirmation of dark energy's existence is through the idea that dark energy is best explained by the "cosmological constant", proposed first by Einstein, which is equivalent to the energy of the vacuum.

Dear reader, after these explanations, I believe that we are ready for my peculiar new hypothesis.

This hypothesis is based on the experimental confirmation, which we just discussed, that dark energy has not changed over billions of years. I expand on this discovery, by proposing the idea that not only dark energy has remained unchanged during the life of our universe, but it also existed at the time of the creation of our universe. And, most importantly, I assume that dark energy WAS the Creator of our universe. In my human imagination, dark energy is an intelligent being with unimaginably powerful intellectual and creative abilities. In my hypothesis, I assume that there are an infinite number of other dark energies, similar to the one in our universe that have created and continue to create other universes.

And now, let's focus on our universe. I have no idea why it was created. I have only great hopes that we, humans, as beings with some basic intelligence, are needed by its Creator. And when it comes to the puzzling values of the physical constants mentioned above, and, for that matter, all the physical laws that govern our universe, those are simply inherent to the intelligent Being (aka the dark energy) that has created the universe and carries it within itself. As for us, humans, we have simply adapted to those constants and laws. And this is the new interpretation of the famous anthropic principle that I propose.

To expand further, I am convinced that there are an infinite number of other intelligent beings in our universe who have come to exist and adapted to live in conditions very different from ours. Those other intelligent beings are, therefore, very different from us, but there is still something that connects us and unites us with them. The uniting link is the intelligence (albeit in different degrees of development), and our common goals, that connect us all with our Creator - the dark energy in our universe. I think that realizing the need to connect with the other intelligent beings that are closest to us is one of the most important priorities we face.

Dear reader, I am firmly convinced that, even at this very moment, we are in contact with other intelligent beings in our universe. However, I believe that this contact, this relationship, is one-sided - on their part it is conscious, and on our part, we are yet to realize it. But I also believe that this realization is about to happen in the very near future.

\section{References}

1. Greene Brian (2011) The Hidden Reality. Alfred A. Knopf, Newyork.

2. Bartlett Richard (2009) The Physics of Miracles. Atria Books.

3. Castaneda Carlos (1993) The Art of Dreaming. HarperCollins Pub, New York.

4. Stephen W Hawking (1973) A Brief History of Time. Bantam Books.

5. Rubin Vera (1997) Bright Galaxies, Dark Matter. American Journal of Physics 65: 1028. 